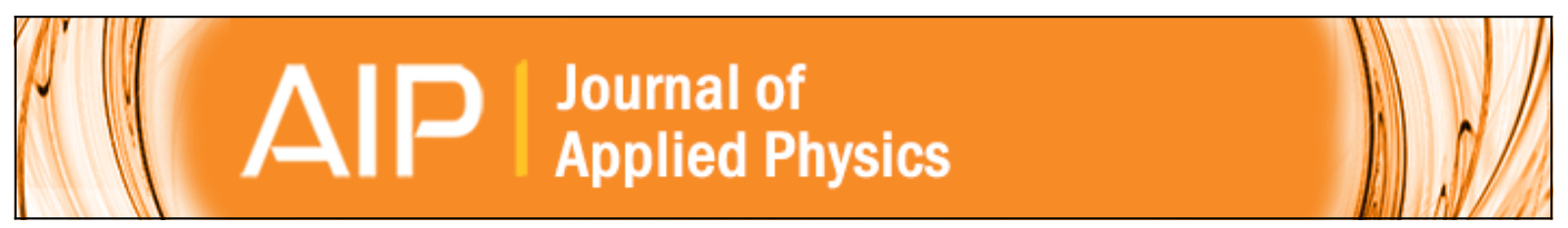

\title{
Cobalt ferrite nanoparticles under high pressure
}

F. D. Saccone, S. Ferrari, D. Errandonea, F. Grinblat, V. Bilovol, and S. Agouram

Citation: Journal of Applied Physics 118, 075903 (2015); doi: 10.1063/1.4928856

View online: http://dx.doi.org/10.1063/1.4928856

View Table of Contents: http://scitation.aip.org/content/aip/journal/jap/118/7?ver=pdfcov

Published by the AIP Publishing

\section{Articles you may be interested in}

Microstructure and magnetic properties of $\mathrm{MFe} 2 \mathrm{O} 4(\mathrm{M}=\mathrm{Co}, \mathrm{Ni}$, and $\mathrm{Mn})$ ferrite nanocrystals prepared using colloid mill and hydrothermal method

J. Appl. Phys. 117, 17A328 (2015); 10.1063/1.4917463

Nanocrystalline Ni-Al ferrites for high frequency applications

AIP Conf. Proc. 1512, 408 (2013); 10.1063/1.4791084

Magnetic and electrical properties of In doped cobalt ferrite nanoparticles

J. Appl. Phys. 112, 084321 (2012); 10.1063/1.4759436

Size effects on structural and magnetic properties of $\mathrm{CoFe} 2 \mathrm{O} 4$ nanoparticles prepared by co-precipitation method

AIP Conf. Proc. 1447, 289 (2012); 10.1063/1.4709993

Lattice preferred orientation and stress in polycrystalline hcp-Co plastically deformed under high pressure J. Appl. Phys. 100, 023510 (2006); 10.1063/1.2214224

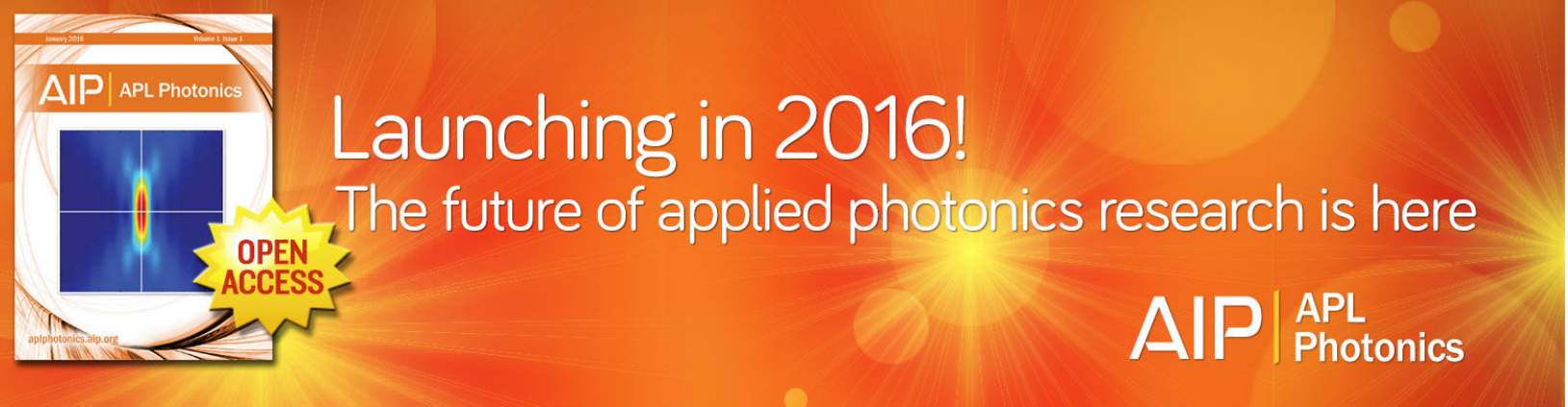




\title{
Cobalt ferrite nanoparticles under high pressure
}

\author{
F. D. Saccone, ${ }^{1}$ S. Ferrari, ${ }^{1}$ D. Errandonea, ${ }^{2, a)}$ F. Grinblat, ${ }^{1}$ V. Bilovol, ${ }^{1}$ and S. Agouram ${ }^{3}$ \\ ${ }^{1}$ Instituto de Tecnologías y Ciencias de la Ingeniería, "Ing. H. Fernández Long," Av. Paseo Colón 850 (1063), \\ Buenos Aires, Argentina \\ ${ }^{2}$ Departamento de Fisica Aplicada, Institut Universitari de Ciència dels Materials, Universitat de Valencia, \\ c/ Doctor Moliner 50, E-46100 Burjassot, Valencia, Spain \\ ${ }^{3}$ Departamento de Física Aplicada y Electromagnetismo, Universitat de València, 46100 Burjassot, Valencia, \\ Spain
}

(Received 22 June 2015; accepted 7 August 2015; published online 20 August 2015)

\begin{abstract}
We report by the first time a high pressure X-ray diffraction and Raman spectroscopy study of cobalt ferrite $\left(\mathrm{CoFe}_{2} \mathrm{O}_{4}\right)$ nanoparticles carried out at room temperature up to $17 \mathrm{GPa}$. In contrast with previous studies of nanoparticles, which proposed the transition pressure to be reduced from 20-27 GPa to 7.5-12.5 GPa (depending on particle size), we found that cobalt ferrite nanoparticles remain in the spinel structure up to the highest pressure covered by our experiments. In addition, we report the pressure dependence of the unit-cell parameter and Raman modes of the studied sample. We found that under quasi-hydrostatic conditions, the bulk modulus of the nanoparticles $\left(\mathrm{B}_{0}=204 \mathrm{GPa}\right)$ is considerably larger than the value previously reported for bulk $\mathrm{CoFe}_{2} \mathrm{O}_{4}$ $\left(\mathrm{B}_{0}=172 \mathrm{GPa}\right)$. In addition, when the pressure medium becomes non-hydrostatic and deviatoric stresses affect the experiments, there is a noticeable decrease of the compressibility of the studied sample $\left(\mathrm{B}_{0}=284 \mathrm{GPa}\right)$. After decompression, the cobalt ferrite lattice parameter does not revert to its initial value, evidencing a unit cell contraction after pressure was removed. Finally, Raman spectroscopy provides information on the pressure dependence of all Raman-active modes and evidences that cation inversion is enhanced by pressure under non-hydrostatic conditions, being this effect not fully reversible. (C) 2015 AIP Publishing LLC. [http://dx.doi.org/10.1063/1.4928856]
\end{abstract}

\section{INTRODUCTION}

Spinel-structured $\mathrm{MT}_{2} \mathrm{O}_{4}$ oxides form part of a very large family of compounds that include more than eighty different oxides. ${ }^{1}$ These oxides are not only widely spread in nature occurring as minerals all over the globe but also are commonly synthesized or grown in laboratories. Their study is relevant for many technological applications and is also of importance for earth and planetary sciences. ${ }^{2}$ The crystal structures of most spinel-type oxides are already known for a long time. ${ }^{3}$ Most of them have a cubic structure (space group $\mathrm{Fd} \overline{3} \mathrm{~m}$, No. 227), which is so simple that can be considered as a textbook example. The classical representation of this crystal structure is $\mathrm{MgAl}_{2} \mathrm{O}_{4} \cdot{ }^{4}$ The cubic spinel structure can be seen as a cubic close-packing of oxygen atoms with $\mathrm{M}$ and $\mathrm{T}$ cations occupying $\frac{1}{2}$ and $\frac{1}{4}$ of the octahedral and tetrahedral sites, respectively. In general, most spinels have some degree of inversion (the mutual substitution of $\mathrm{M}$ and T cations). ${ }^{2}$ Cobalt ferrite $\left(\mathrm{CoFe}_{2} \mathrm{O}_{4}\right)$ usually exhibits an intermediate degree of inversion, which depends on the preparation method. ${ }^{5}$

After Finger et $a l .{ }^{6}$ studied the structure of spinel and magnetite under compression up to $4 \mathrm{GPa}$, spinel-type oxides have been the focus of high-pressure (HP) studies. ${ }^{2,5,7-10}$ In particular, HP x-ray diffraction (XRD) experiments have been performed recently to study the crystal structure of cobalt ferrite. ${ }^{5,7}$ It has been reported that the onset of a phase transition to a post-spinel structure takes place at around $20-27 \mathrm{GPa} .^{5,7}$

\footnotetext{
a) Author to whom correspondence should be addressed. Electronic mail: daniel.errandonea@uv.es
}

In addition, the bulk modulus $\left(\mathrm{B}_{0}\right)$ of $\mathrm{CoFe}_{2} \mathrm{O}_{4}$ has been determined, being found that its value depends largely on the experimental conditions. From quasi-hydrostatic experiments performed up to $10 \mathrm{GPa}, \mathrm{B}_{0}=175 \mathrm{GPa}$ is obtained. ${ }^{7}$ This value agrees with the bulk modulus reported from quasihydrostatic experiments carried out up to $25 \mathrm{GPa} .^{5}$ From not hydrostatic experiments up to $23 \mathrm{GPa}, \mathrm{B}_{0}=250 \mathrm{GPa}$ is obtained. ${ }^{7}$ These values of $\mathrm{B}_{0}$, however, are considerably larger than the value reported for the tetragonal phase of $\mathrm{CoFe}_{2} \mathrm{O}_{4}\left(\mathrm{~B}_{0}=94 \mathrm{GPa}\right)$, a distorted version of cubic spinel. ${ }^{11}$ This fact is in contradiction with the results reported for spinel $\mathrm{ZnGa}_{2} \mathrm{O}_{4}$ for which the compression of the cubic and tetragonal polymorphs can be described with the same equation of state (EOS). ${ }^{9}$ On top of that, HP resistivity experiments carried out in $\mathrm{CoFe}_{2} \mathrm{O}_{4}$ nanocrystals indicated that the phase transition to the post-spinel structure occurs at 7.5 and $12 \mathrm{GPa}$ for 80 and $6 \mathrm{~nm}$ particles, respectively. ${ }^{12}$ The reduction of the transition pressure from $20 \mathrm{GPa}$ to less than $12 \mathrm{GPa}$ was explained as a consequence of the transformation of $\mathrm{CoFe}_{2} \mathrm{O}_{4}$ into a metastable phase that does not exist in the bulk material. ${ }^{12}$ However, this result contradicts the known fact that transition pressure usually shifts towards higher pressures when reducing the size of the nanocrystal. ${ }^{13}$

Regarding HP Raman experiments on $\mathrm{CoFe}_{2} \mathrm{O}_{4}$, they have been only reported for the tetragonal polymorph. ${ }^{11}$ This and all the facts described above suggest that it is timely to perform additional high-pressure studies on $\mathrm{CoFe}_{2} \mathrm{O}_{4}$. Here, to contribute to the understanding of the high-pressure behavior of $\mathrm{CoFe}_{2} \mathrm{O}_{4}$, we report a synchrotron x-ray diffraction and Raman spectroscopy study of $\mathrm{CoFe}_{2} \mathrm{O}_{4}$ 
nanoparticles up to $17 \mathrm{GPa}$. It is a well-known fact that in nanocrystals, both transition pressures and properties like the bulk modulus could depend upon the particle size. ${ }^{14}$ In addition to particle size, there are other facts that could influence the HP behavior of nanocrystals; one is the selection of the pressure-transmitting medium (PTM) used in high-pressure experiments, which may have a strong influence on the physical state of the studied sample. ${ }^{15-17}$ Therefore, to compare with the most recent HP XRD study, ${ }^{7}$ we performed our experiments using the same PTM. However, to compare with the previous studies on $\mathrm{CoFe}_{2} \mathrm{O}_{4}$ nanoparticles ${ }^{11}$ and to reduce the influence of non hydrostaticity, we limit the maximum pressure to $17 \mathrm{GPa}$. We found that no pressure-induced phase transition takes place in $\mathrm{CoFe}_{2} \mathrm{O}_{4}$, which contradicts resistivity studies. ${ }^{12}$ We also observed in $\mathrm{CoFe}_{2} \mathrm{O}_{4}$ nanoparticles, a Hall-Petch strengthening, a decrease of compressibility under non hydrostatic conditions, and an evidence that cation inversion is enhanced by pressure.

\section{EXPERIMENTAL}

Iron(III) chloride hexahydride $\left(\mathrm{FeCl}_{3} \cdot 6 \mathrm{H}_{2} \mathrm{O}\right.$, Tetrahedron) and cobalt(II) chloride hexahydride $\left(\mathrm{CoCl}_{2} \cdot 6 \mathrm{H}_{2} \mathrm{O}, 98 \%\right.$, Cicarelli) were used to synthesize $\mathrm{CoFe}_{2} \mathrm{O}_{4}$ nanoparticles by co-precipitation method in a $\mathrm{NaOH}$ medium, keeping the molar ratio of $\mathrm{Co} / \mathrm{Fe}=0.5$. Each ingredient was dissolved in a $\mathrm{NaOH}$ solution and then the solutions were mixed. The digestion was performed at $80^{\circ} \mathrm{C}$ for $120 \mathrm{~min}$. After digestion, the gelatinous precipitate was filtered and washed several times using deionized water until the $\mathrm{pH}$ value of the solution became neutral. Finally, the gelatinous precipitate was dried at room temperature (RT) in air to obtain a powder sample. Annealing of the powder was performed at $700{ }^{\circ} \mathrm{C}$ during $10 \mathrm{~h}$.

The morphological and structural characterization of the samples was performed by transmission electron microscopy (TEM), high-resolution TEM (HRTEM), and selected area electron diffraction (SAED) by using a FEI Field Emission Gun (TECNAI G2 F20 S-TWIN) microscope operated at $200 \mathrm{kV}$. Energy-dispersive x-rays spectroscopy (EDXS) in TEM nanoprobe mode was achieved to check the purity of the synthesized nanoparticles. To perform the measurement, the samples were treated by sonicating in absolute ethanol for few minutes. Then, a drop of the resulting suspension was deposited onto a holey-carbon film supported on a copper grid, which was subsequently dried.

Ambient pressure structural characterization and phase identification of the nanopowder was carried out by XRD with a Rigaku D/max diffractometer equipped with a vertical goniometer, using a Bragg-Brentano geometry ( $\theta-2 \theta$ coupled arms) and monochromatic $\mathrm{Cu}-\mathrm{K}_{\alpha}$ radiation in the $15^{\circ} \leq 2 \theta \leq 100^{\circ}$ range, measuring at every $0.05^{\circ}$ step and sweeping with a $0.4^{\circ}$ per minute velocity. To determine cation inversion, Mössbauer measurements were recorded at RT under transmission geometry with a standard constant acceleration spectrometer, using a $5 \mathrm{mCi}{ }^{57} \mathrm{CoRh}$ radioactive source for ${ }^{57} \mathrm{Fe}$ Mössbauer studies. Data were recorded using a 1024 channel MDAQ107 data acquisition module. ${ }^{18}$
HP powder diffraction experiments were performed at the XDS beam-line of Laboratorio Nacional de Luz Sincrotron (LNLS), Campinas, Brazil. Pressure was applied by means of a membrane diamond-anvil cell (DAC), being measured the samples under compression and decompression. The applied pressure was determined by the ruby fluorescence method with an accuracy of $0.1 \mathrm{GPa}^{19}$ A $4: 1$ methanol-ethanol mixture was used as PTM. ${ }^{20}$ Special attention was paid during sample loading into the DAC to avoid sample overloading, which leads to sample bridging under compression affecting the measurements. ${ }^{16}$ The experiments were performed in the angle-dispersive configuration with a monochromatic beam with wavelength of 0.620231(5) A. The images were collected using a CCD Rayonix 165. The structural analysis was performed using MAUD. ${ }^{21}$

Raman studies were performed using a DAC. Samples were loaded under identical conditions than in XRD measurements, and pressure was measured using the ruby scale. ${ }^{19}$ Raman experiments were carried out in backscattering geometry with a Jobin-Yvon single spectrometer equipped with an edge filter and a thermoelectric-cooled multichannel CCD detector. Measurements with a spectral resolution of $1 \mathrm{~cm}^{-1}$ were performed using the $514.5 \mathrm{~nm}$ line of an $\mathrm{Ar}$ laser. Laser power was kept below $20 \mathrm{~mW}$ to avoid sample heating.

\section{RESULTS AND DISCUSSIONS}

\section{A. Sample characterization}

The TEM micrograph presented in Fig. 1(a) shows an image of the $\mathrm{CoFe}_{2} \mathrm{O}_{4}$ sample. Fig. 1(b) shows the corresponding SAED pattern. We found that the sample consists of small grains with size ranging from 10 to $50 \mathrm{~nm}$ in diameter. The major part of grains has a size of 10-20 nm. The SAED pattern (Fig. 1(b)) exhibits at least six well defined diffraction ring characteristics of a polycrystalline nature of the $\mathrm{CoFe}_{2} \mathrm{O}_{4}$ nanoparticles. The concentric rings are produced by the $\mathrm{CoFe}_{2} \mathrm{O}_{4}$ nanoparticles randomly distributed giving a continuous angular distribution of (hkl) spots. The different rings can be indexed with the cubic spinel structure of $\mathrm{CoFe}_{2} \mathrm{O}_{4}$ (JCPDS card 22-1086) with space group Fd $\overline{3} \mathrm{~m}$. The interplanar distances (d-values) determined from the radius of the rings are $2.945,2.492,2.087,1.595,1.472$, and $1.270 \AA$, corresponding to the planes (220), (311), (400), (511), (440), and (533), respectively. It is important to note that no obvious rings corresponding to other compounds were observed in SAED patterns indicating that the obtained nanoparticles are pure $\mathrm{CoFe}_{2} \mathrm{O}_{4}$ phase products. This fact was confirmed by TEM-EDXS measurements. In the EDXS spectrum shown in Fig. 2(b), in addition to $\mathrm{Co}, \mathrm{Fe}$, and $\mathrm{O}$, only $\mathrm{C}$ and $\mathrm{Cu}$ (present in the sample holder) can be detected. The measured Co and Fe content was about 33.5(5) at. \% and 66.5(5) at. \%, respectively, confirming that the nanoparticles are composed of $\mathrm{CoFe}_{2} \mathrm{O}_{4}$.

The single crystalline structure of nanoparticles was also confirmed by high-resolution TEM images, as shown in Fig. 2(a), suggesting that the nanoparticles were single crystals as indicated clearly by atomic lattice fringes. Direct measurement of spacing in between the crystal fringes visualized in 

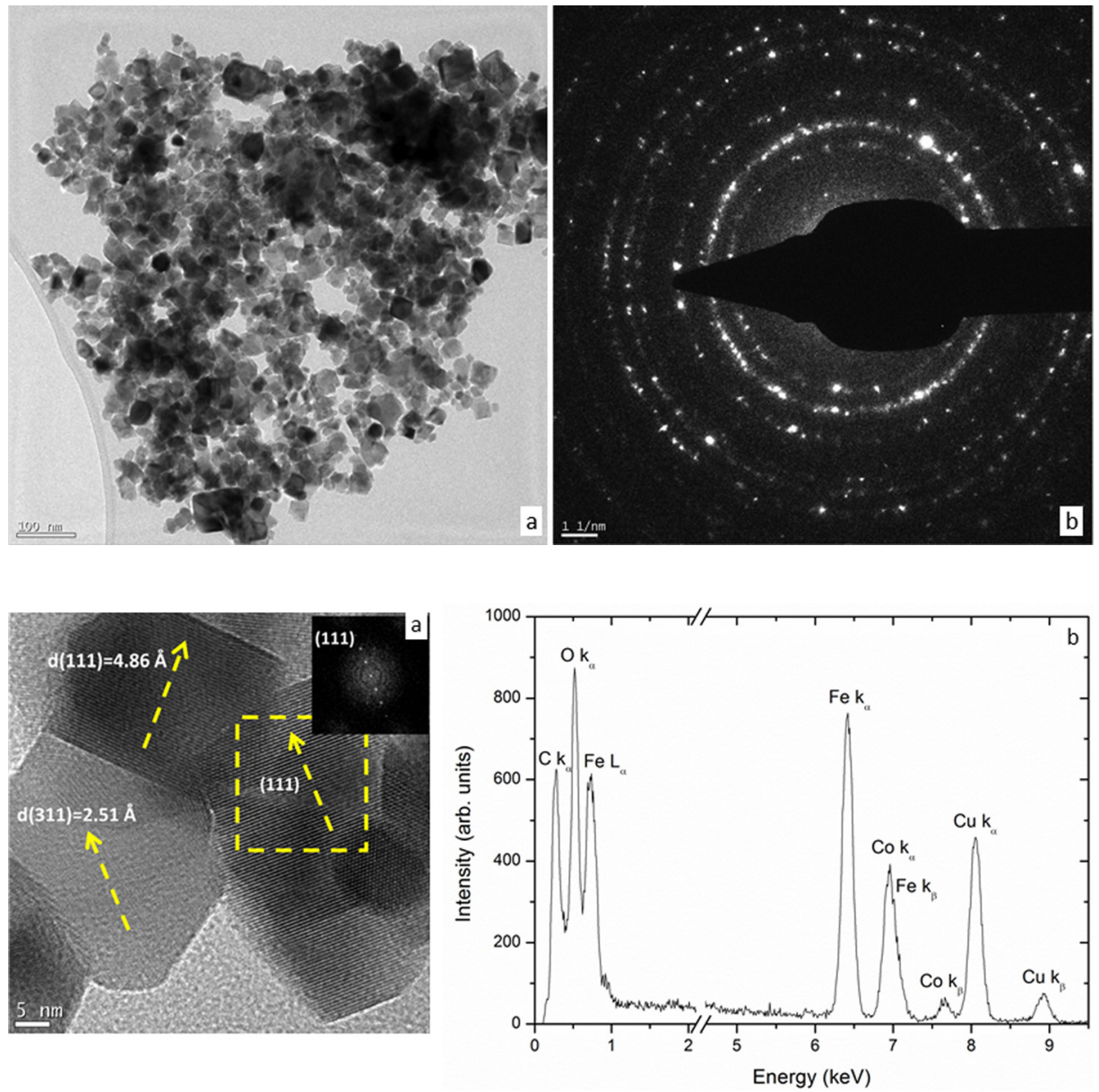

FIG. 1. (a) TEM micrograph of the $\mathrm{CoFe}_{2} \mathrm{O}_{4}$ nanoparticles prepared. (b) SAED pattern of the same sample.
FIG. 2. (a) High-resolution TEM image. (b) EDXS spectrum of the $\mathrm{CoFe}_{2} \mathrm{O}_{4}$ nanoparticles. the HRTEM micrograph is about $4.86 \AA$ (Fig. 2(a)) corresponding to the (111) lattice spacing of $\mathrm{CoFe}_{2} \mathrm{O}_{4}$. In addition, the lattice spacing of $2.51 \AA$ (Fig. 2(a)) corresponds to the (311) planes of $\mathrm{CoFe}_{2} \mathrm{O}_{4}$. A fast Fourier transformation of the selected zone confirmed the monocrystalline structure of the selected nanoparticle. Local EDXS analysis in nanoprobe mode (spot size of the beam $<5 \mathrm{~nm}$ ) confirmed the composition of the nanoparticles.

The XRD pattern measured at ambient pressure is shown in Fig. 3. It is seen that the sample is in crystalline state and monophasic with a cubic spinel structure similar to JCPDS card 22-1086. We determined from Rietveld analysis a lattice constant of 8.3780(2) $\AA$. We also determined the oxygen position to be $(0.2505(2), 0.2505(2), 0.2505(2))$. These values are in agreement with the results reported by Baraliya and Joshi ${ }^{22}$ and Kumar et al. ${ }^{23}$ The lattice constant is $1 \%$ larger than in the bulk material, which is a typical feature of $\mathrm{CoFe}_{2} \mathrm{O}_{4}$ nanoparticles. $^{22,23}$ However, using the Scherrer's equation, ${ }^{24}$ from the full width at hall maximum (FWHM) of peak (311), a mean grain size of 38(2) nm is obtained. Fig. 3 also shows the result of the Rietveld refinement. It can be seen that the powder XRD pattern can be unequivocally assigned to the cubic spinel structure. In the Rietveld refinement, the oxygen position $(\mathrm{x}, \mathrm{x}, \mathrm{x})$ has been taken as free parameter. All other fractional positions are fixed by symmetry. Other parameters such as unit-cell parameters, isothermal parameters, scale factors, and shape parameters have been considered as free parameters. The occupation of the oxygen positions was fixed to be 1 based upon stoichiometry, and the occupation of the cationic sites was assumed to be the one obtained from the Mössbauer measurements described below. This assumption was taken because of the similar x-ray scattering factor of Co and Fe, which precludes the accurate determination of the degree of cation inversion in $\mathrm{CoFe}_{2} \mathrm{O}_{4}$ from powder XRD experiments.

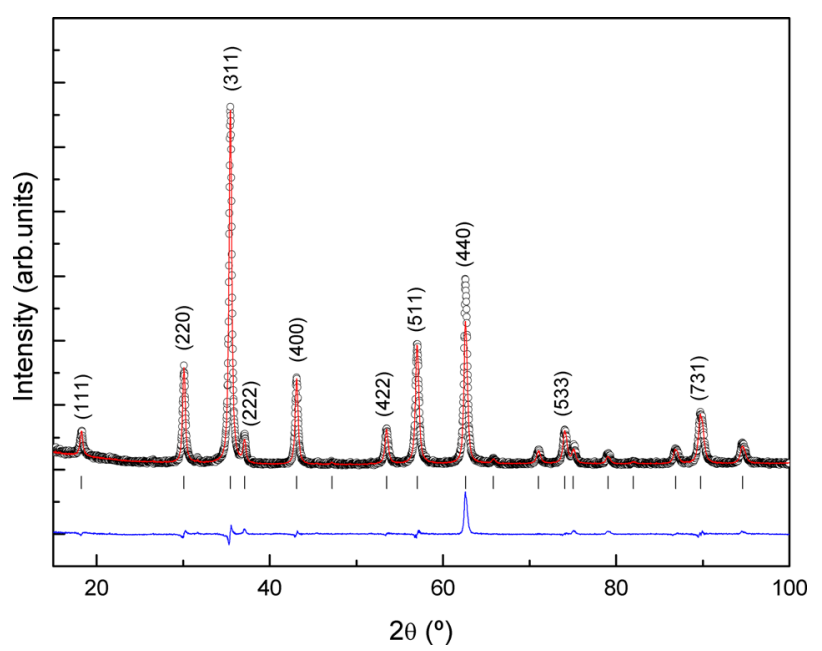

FIG. 3. Ambient pressure x-ray diffraction pattern of $\mathrm{CoFe}_{2} \mathrm{O}_{4}\left(\mathrm{Cu} \mathrm{K} \mathrm{K}_{\alpha}\right.$ radiation). Symbols represent the experiments. The red solid line shows the calculated profile. The blue solid line shows the residual of the refinement. Ticks indicated the calculated Bragg reflections. The indexes of the most representative peaks of the cubic spinel structure are indicated. 
The background has been corrected using a 5th order polynomic function. The goodness-of-fit parameters of the refinement are $\mathrm{R}_{\mathrm{W}}=14.96 \%, \mathrm{R}_{\mathrm{B}}=10.12 \%$, and $\chi^{2}=2.24$.

The ${ }^{57} \mathrm{Fe}$ Mössbauer spectrum for the cobalt ferrite nanoparticle powders is shown in Fig. 4, where the existence of three sextets with magnetic hyperfine field of $44.4 \mathrm{~T}$, $48.4 \mathrm{~T}$, and 50.9 T, respectively, can be observed. The two sextets with higher hyperfine fields can be related to octahedral sites, or B sites, and the first one (with the lowest hyperfine field) is the corresponding to tetrahedral site, or A site. Likewise, it was needed to add a quadrupole doublet as a minority site to explain the measured spectrum. The A/B occupation ratio was determined from comparison of sextet areas, as being $\mathrm{A} / \mathrm{B}=0.248$, which corresponds to a lower fraction of ${ }^{57} \mathrm{Fe}$ probe occupying A sites. Then, it can be concluded that from our synthesis, we obtained mixed cobalt ferrite spinel as the only phase.

\section{B. X-ray powder diffraction at high-pressure}

In Fig. 5, we show a selection of HP XRD patterns. The observed peaks correspond to $\mathrm{CoFe}_{2} \mathrm{O}_{4}$ spinel structure. At some pressures, we observe extra peaks (depicted by $*$ in the figure), which corresponds to Bragg peaks of the ruby used as pressure scale. No evidence of phase transition is found up to $13 \mathrm{GPa}$. Even the tetragonal distortion of the cubic spinel structure, observed in other spinels under compression, ${ }^{9}$ is not observed in our experiments. This fact contradicts the conclusions extracted from resistivity measurements. ${ }^{12}$ In addition, no change in the relative intensity of spinel Bragg peaks is detected within the accuracy of the experiments up to the highest pressure achieved. This suggests that internal atomic positions are slightly affected by pressure up to $13 \mathrm{GPa}$. If this were not the case, then the relative intensity of the (311) and (400) reflections should change considerable, ${ }^{25}$ which is not observed in the present experiments.

From the measured XRD patterns, we obtained the pressure dependence for the unit-cell parameter and volume of $\mathrm{CoFe}_{2} \mathrm{O}_{4}$. This information was obtained from Rietveld refinements. The procedure used for the refinements was the same used at ambient pressure with the only difference that the atomic position of oxygen was fixed to the ambient pressure position. This was done to reduce the number of free

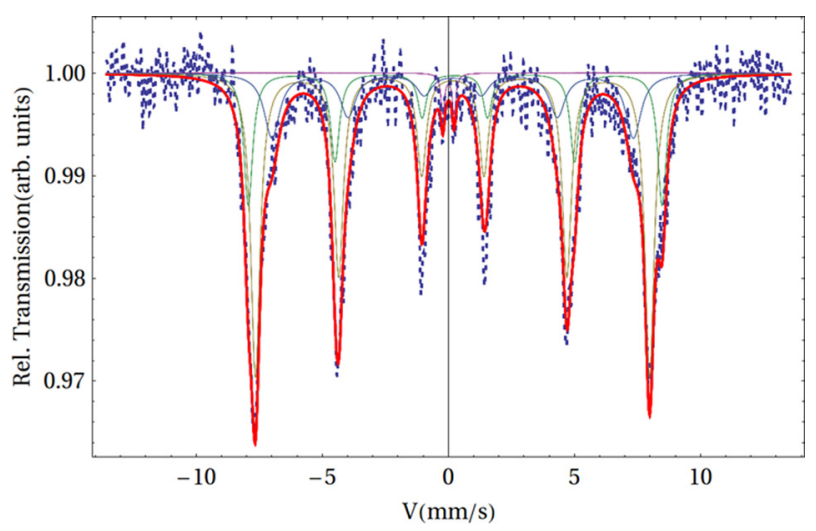

FIG. 4. Room temperature Mössbauer spectrum (and fitting) measured from $\mathrm{CoFe}_{2} \mathrm{O}_{4}$ nanoparticles. Dots: experiment.

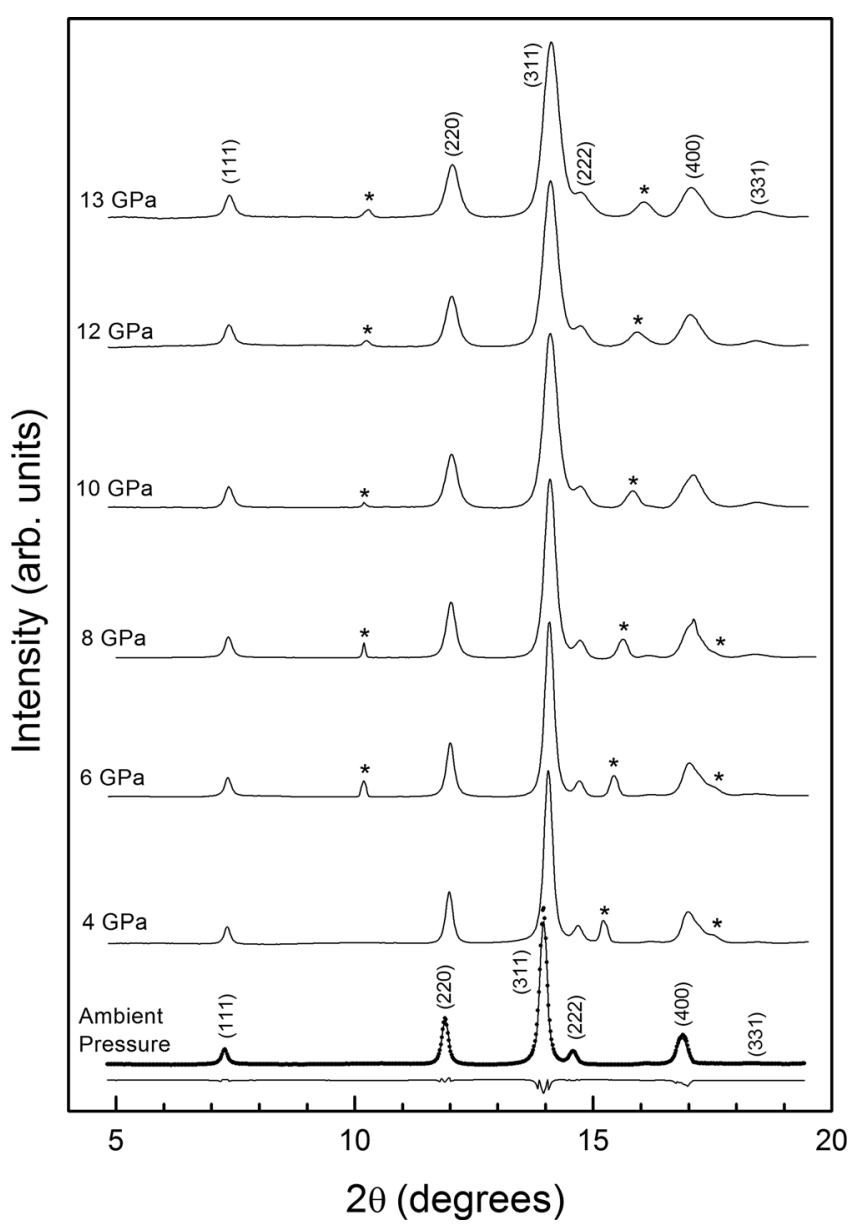

FIG. 5. Synchrotron XRD patterns measured in $\mathrm{CoFe}_{2} \mathrm{O}_{4}$ at different pressures. At ambient pressure, the dots represent the experiment at the solid line the Rietveld refinement and the residuals. The peaks of the spinel structure of $\mathrm{CoFe}_{2} \mathrm{O}_{4}$ are labeled. The symbol * is used to identify peaks of the ruby used to measure pressure.

parameters because less Bragg peaks were measured under compression due to the angular constrain imposed by the $\mathrm{DAC}$ and the CCD detector. This assumption is reasonable based on the discussion we did on relative peak intensity in the previous paragraph. This is common assumption in HP studies and will not affect the determination of the unit-cell parameter. ${ }^{26}$ In Fig. 5, we show the residual of the refinement of the XRD experiment carried out at ambient pressure within the DAC. Similar residuals were obtained at all pressures. The small residuals indicate that the assumed structural model is reasonable. The goodness-of-fit values of the refinement shown in the figure are $\mathrm{R}_{\mathrm{W}}=3.76 \%, \mathrm{R}_{\mathrm{B}}=5.12 \%$, and $\chi^{2}=1.92$. The obtained unit-cell parameters versus pressure are given in Table I.

The unit-cell volume compression is shown in Fig. 6. It is compared with the results recently reported for bulk $\mathrm{CoFe}_{2} \mathrm{O}_{4}{ }^{7}$ A first conclusion that can be extracted is that in the nanoparticle experiment at $8 \mathrm{GPa}$, there is a reduction of the compressibility. This pressure is comparable with the pressure limit for the quasi-hydrostatic behavior of the PTM, which become frozen about $8 \mathrm{GPa}^{20}$ As a consequence of it, deviatoric stresses affect significantly the results ${ }^{15-17}$ producing a behavior that is not intrinsic. The relevance of deviatoric 
TABLE I. Unit-cell parameter (a) at different pressures determined from DAC experiments. The error in pressure is $0.1 \mathrm{GPa}$. The error of $a$ is indicated in the table.

\begin{tabular}{lcccc}
\hline \hline \multirow{2}{*}{ Compression } & & & \multicolumn{2}{c}{ Decompression } \\
$\mathrm{P}(\mathrm{GPa})$ & $a(\AA)$ & & $\mathrm{P}(\mathrm{GPa})$ & $a(\AA)$ \\
\hline 0 & $8.378(4)$ & & 0 & $8.348(4)$ \\
2 & $8.343(4)$ & & 2 & $8.329(4)$ \\
4 & $8.318(4)$ & & 3 & $8.320(4)$ \\
6 & $8.303(4)$ & & 4 & $8.313(4)$ \\
8 & $8.287(4)$ & & 5 & $8.299(4)$ \\
10 & $8.279(4)$ & & 6 & $8.290(4)$ \\
12 & $8.275(4)$ & & 10 & \\
13 & $8.269(4)$ & & & \\
\hline \hline
\end{tabular}

stresses above $8 \mathrm{GPa}$ can be clearly seen in Fig. 7. This figure illustrates the broadening of XRD peaks that takes place above $8 \mathrm{GPa}$, a clear evidence that deviatoric stresses are not negligible beyond $8 \mathrm{GPa}$. Then, we can conclude that non hydrostaticity reduces the compressibility of $\mathrm{CoFe}_{2} \mathrm{O}_{4}$, which is in complete agreement with the recent report of Blasco et $a .^{7}$ as can be seen in Fig. 6. Another conclusion that can be extracted from Fig. 6 is that after decompression (empty symbols), there is a hysteresis in the pressure dependence of the unit-cell volume, having the recovered sample a unit-cell volume smaller than the as-sintered nanoparticle powder.

The pressure-volume data measured in the pressure range, where deviatoric stresses were found to be negligible $(\mathrm{P}<8 \mathrm{GPa})$, were analyzed using a second-order BirchMurnaghan EOS, ${ }^{27}$ employing EOSfit. ${ }^{28}$ Since only four data points were measured upon compression below $8 \mathrm{GPa}$, the unit-cell volume at ambient pressure was fixed to the value measured in the experiment carried out outside the DAC. Therefore, $B_{0}$ was the only fitting parameter, being

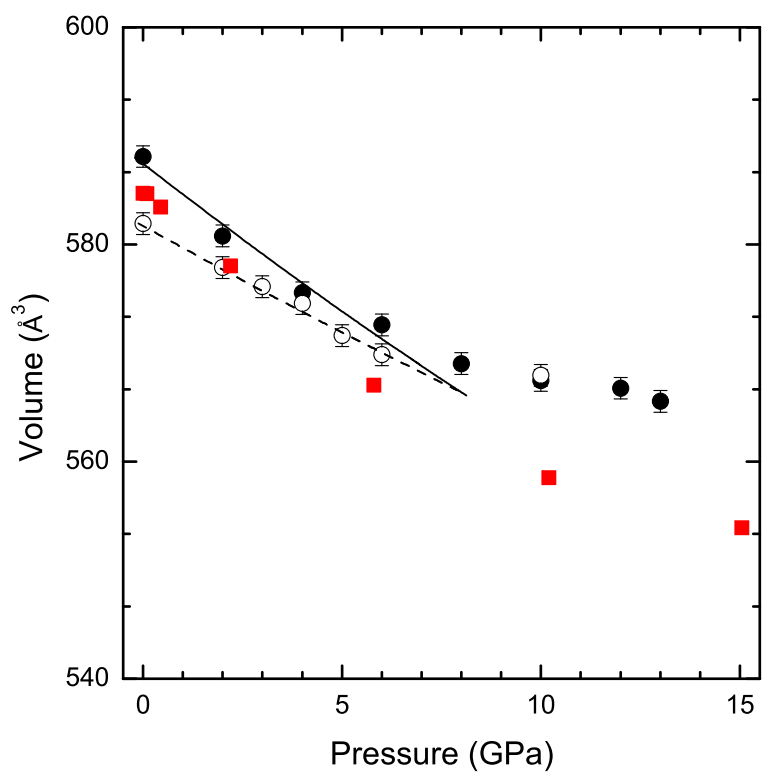

FIG. 6. Unit-cell volume versus pressure. The solid (empty) circles represent data measured under compression (decompression). The red solid squares are results from Ref. 7. The solid (dashed) line shows the EOS fitted under quasi-hydrostatic pressure ( $\mathrm{P}<8 \mathrm{GPa}$ ) for compression (decompression).

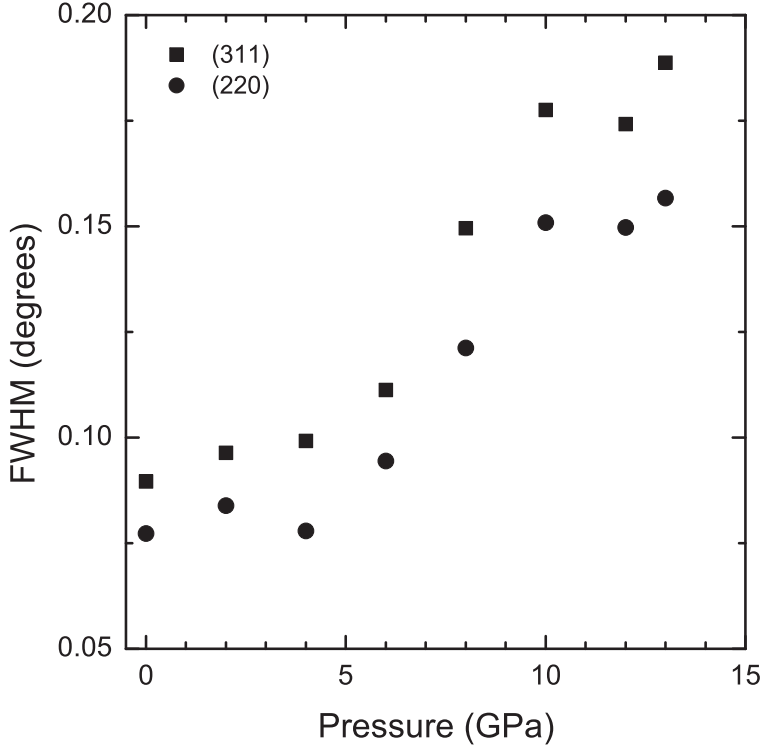

FIG. 7. Full width at half maximum of the (311) and (220) peaks of spinel $\mathrm{CoFe}_{2} \mathrm{O}_{4}$.

the obtained value $\mathrm{B}_{0}=204(12) \mathrm{GPa}$. The weighted $\chi^{2}$ of the fit is 1.1 , being the largest $\Delta \mathrm{P} 0.5 \mathrm{GPa}$. A second-order EOS was used by Blasco et al. ${ }^{7}$ to fit their results. Therefore, the $\mathrm{B}_{0}$ determined here for the nanoparticles can be directly compared with the $\mathrm{B}_{0}$ previously obtained for the bulk material. ${ }^{7}$ Our $B_{0}$ is notably higher than the observed value for a bulk material of a similar composition, $\mathrm{B}_{0}=175(2) \mathrm{GPa}$. Thus, we can conclude that a Hall-Petch strengthening ${ }^{29}$ occurs in $\mathrm{CoFe}_{2} \mathrm{O}_{4}$ nanoparticles. Besides, we found that if results measured above $8 \mathrm{GPa}$ are included in the EOS fit, a second-order EOS cannot explain the compressibility change we found at $8 \mathrm{GPa}$. In such a case, if a third-order EOS is used, we obtain an unusual large value for pressure derivative of the bulk modulus, $\mathrm{B}_{0}{ }^{\prime}=17(5)$ $\mathrm{GPa}$. On the other hand, if the determined 2nd order EOS is extrapolated to pressures higher than $8 \mathrm{GPa}$, the unit-cell volume is underestimated in comparison with experiments (see Fig. 6). Both facts indicate that the compressibility change that occurs when the deviatoric stresses become noticeable (leading to a non-intrinsic behavior) cannot be properly described by the Birch-Murnaghan EOS. This is a common phenomenon observed in many compounds. ${ }^{30,31}$ Therefore, only data measured under quasi-hydrostatic conditions should be used to determine the bulk modulus from compression studies. In our case, if only the results measured under non-hydrostatic conditions are fitted with a second-order EOS, $\mathrm{B}_{0}=284(25) \mathrm{GPa}$ is obtained. The "increase" of the bulk modulus triggered by deviatoric stresses is comparable to the one observed in bulk $\mathrm{CoFe}_{2} \mathrm{O}_{4}{ }^{7}$ In our nanoparticles, $\mathrm{B}_{0}$ goes from 204 to $284 \mathrm{GPa}(40 \%$ increase) and in the bulk material from 175 to $250 \mathrm{GPa}$ (43\% increase).

A final comment we would like to make on the EOS of $\mathrm{CoFe}_{2} \mathrm{O}_{4}$ is that our work and two previous works ${ }^{5,7}$ give values for $\mathrm{B}_{0}$ that exceeds by $100 \%$ the bulk modulus reported for tetragonal spinel $\mathrm{CoFe}_{2} \mathrm{O}_{4}\left(\mathrm{~B}_{0}=94 \mathrm{GPa}\right) .{ }^{11}$ This fact is quite unusual since commonly cubic and tetragonal 
spinels have similar compressibilities. ${ }^{2,9}$ For example, tetragonal spinel $\mathrm{MgMn}_{2} \mathrm{O}_{4}$ has $\mathrm{B}_{0}=156(0.7) \mathrm{GPa}^{32}$ We think that the unusual small bulk modulus reported for the tetragonal $\mathrm{CoFe}_{2} \mathrm{O}_{4}$ (Ref. 11) could probably be hindered by the experimental method. First, experiments were carried out without PTM in Ref. 11. Second, the energy-dispersive XRD patterns shown in Ref. 11 have a very poor resolution, which could influence not only the determination of unit-cell parameters in tetragonal $\mathrm{CoFe}_{2} \mathrm{O}_{4}$ but also pressure determination because Pt Bragg peaks were used to measure pressure. To conclude this point, we would like to note that the EOS given in Ref. 11 deviates from the experimental pressurevolume data points reported in the article. In particular, at $\mathrm{P}=10$ (20) GPa, a volume of 628 (587) $\AA$ is calculated with the reported EOS, which underestimates the experimental volume. A bulk modulus larger than $94 \mathrm{GPa}$ is needed to properly reproduce the experimental results of Ref. 11. This suggests that new studies on tetragonal $\mathrm{CoFe}_{2} \mathrm{O}_{4}$ are needed to determine the behavior of its crystal structure under compression.

\section{High-pressure Raman measurements}

Fig. 8 shows Raman spectra measured at different pressures. We have identified six Raman-active modes at ambient pressure. They agree with those reported by Chandramohan et al ${ }^{33}$ for nanoparticles of similar size than our $\mathrm{CoFe}_{2} \mathrm{O}_{4}$ particles. Raman frequencies from both experiments are compared in Table II. According to group theory, the cubic spinel phase has five Raman-active modes with symmetries: $\Gamma=\mathrm{A}_{1 \mathrm{~g}}+\mathrm{E}_{\mathrm{g}}+3 \mathrm{~T}_{1 \mathrm{~g}}$. However, it is wellknown that due to cation inversion, the highest frequency $\mathrm{A}_{1 \mathrm{~g}}$ modes split into two modes. ${ }^{33,34}$ Consequently, six Raman modes are expected from our sample as we found (see Fig. 8). To avoid confusion, we will label the mode appearing because of cation inversion as $\mathrm{A}_{1 \mathrm{~g} *}$. The six modes have been assigned as shown in Fig. 8 and Table II following the literature. ${ }^{33}$ As pressure increases, we observed several changes in the Raman spectra. All Raman modes harden under compression. In addition, beyond 7.8 GPa, the Raman peaks broaden, changing the FWHM from approximately less than $20 \mathrm{~cm}^{-1}$ to around $25 \mathrm{~cm}^{-1}$. This fact also evidences that deviatoric stresses become not negligible at this pressure, which agrees with the conclusion we extracted from XRD experiments. Fig. 9 shows the pressure dependence of the Raman frequencies. There it can be seen that most modes show a slope change in their pressure evolution beyond $7.8 \mathrm{GPa}$. This could be also a consequence of the increase of deviatoric stresses. The pressure dependences of all phonons below 7.8 GPa and above this pressure could be well fit with different linear functions. The obtained pressure coefficients are given in Table II. There it can be seen that the slope of most modes is reduced beyond 7.8 GPa. This effect is most evident in the $\mathrm{E}_{\mathrm{g}}$ mode with wavelength close to $300 \mathrm{~cm}^{-1}$. The Grüneisen parameter $\gamma=$ $\frac{B_{0}}{\omega_{0}} \frac{\partial \omega}{\partial P}$ calculated for the low-pressure region is given also in Table II. In the equation, $\omega_{0}$ is the frequency at ambient pressure, as given in Table $\mathrm{I}$, and $\mathrm{B}_{0}$ was assumed to be $204 \mathrm{GPa}$.

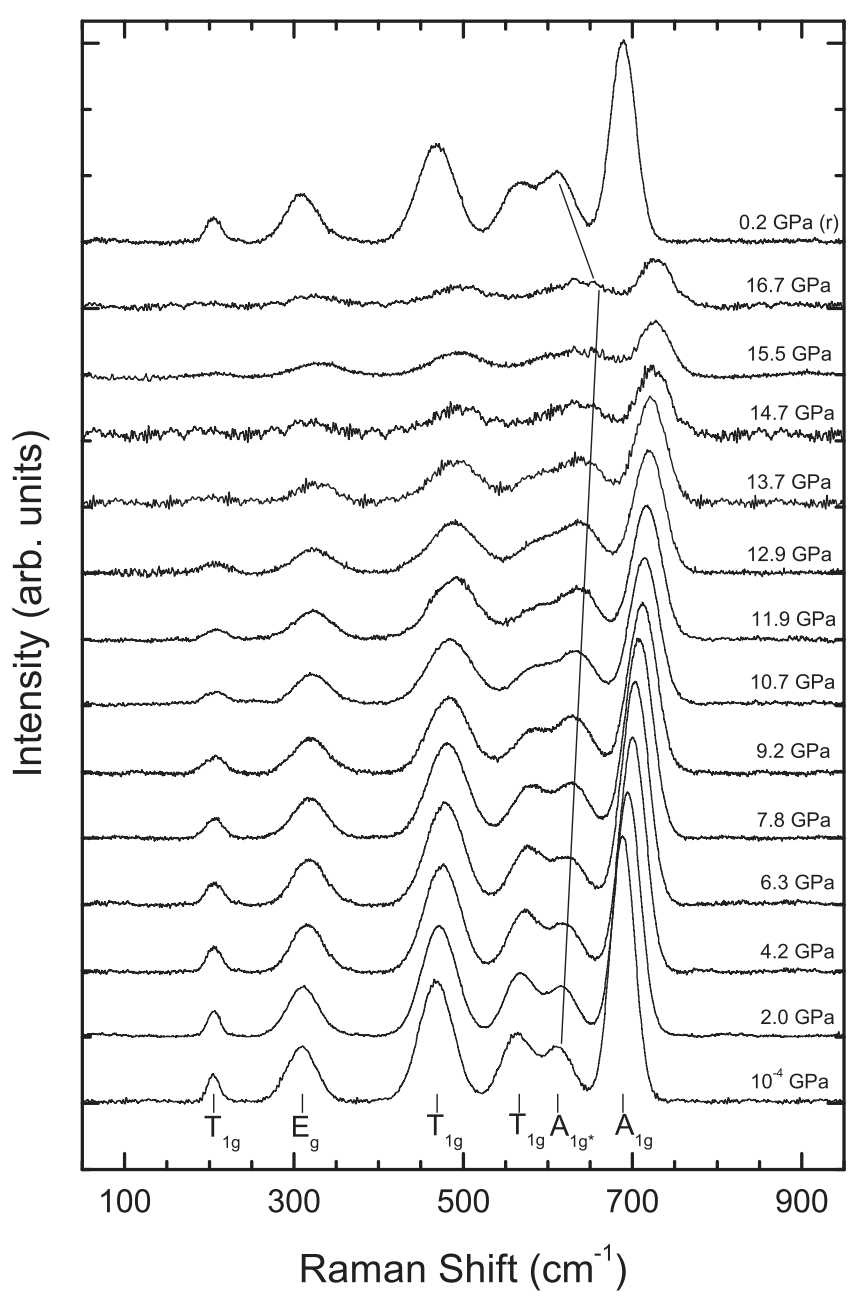

FIG. 8. Raman spectra of $\mathrm{CoFe}_{2} \mathrm{O}_{4}$ nanoparticles at different pressures. Modes are labeled at ambient pressure $\left(10^{-4} \mathrm{GPa}\right)$ and their positions are identified by ticks. The top spectrum denoted by $(\mathrm{r})$ has been collected after pressure release. The solid line show the evolution of the $\mathrm{A}_{1 \mathrm{~g}^{*}}$ mode.

Another fact that we found in the Raman spectra is that the relative intensity between the $\mathrm{A}_{1 \mathrm{~g}}$ and $\mathrm{A}_{1 \mathrm{~g}^{*}}$ modes changes gradually with pressure. This fact is an evidence of the increase of the cation inversion with pressure. In order to see how pressure affects cation inversion, we have plot in Fig. $10 x_{A / B}=\frac{I_{A 1 g^{*}}}{I_{A 1 g}+I_{A 1 g^{*}}}, 35$ where $\mathrm{I}_{\mathrm{A} 1 \mathrm{~g}}$ is the intensity of the Raman mode located near $690 \mathrm{~cm}^{-1}$ and where $\mathrm{I}_{\mathrm{A} 1 \mathrm{~g}^{*}}$ is the intensity of the Raman mode located near $615 \mathrm{~cm}^{-1}$

TABLE II. Frequencies $(\omega)$ of Raman modes and pressure coefficients (d $\omega /$ $\mathrm{dP})$. The Grüneisen parameter $(\gamma)$ is also included. Frequencies are compared with frequencies $\left(\omega_{\mathrm{a}}\right)$ reported in Ref. 33 for 28-nm size nanoparticles. Errors for wavenumbers and Grüneisen parameters are given in the table. The error estimated for $\mathrm{d} \omega / \mathrm{dP}$ is $0.01 \mathrm{~cm}^{-1} / \mathrm{GPa}$.

\begin{tabular}{lccccc}
\hline \hline & $\begin{array}{c}\omega_{\mathrm{a}} \\
\text { Mode }\end{array}$ & $\begin{array}{c}\omega \\
\left(\mathrm{cm}^{-1}\right)\end{array}$ & $\begin{array}{c}\mathrm{d} \omega / \mathrm{dP}\left(\mathrm{cm}^{-1}\right) \\
\mathrm{P}<7.8 \mathrm{GPa})\end{array}$ & $\begin{array}{c}\mathrm{d} \omega / \mathrm{dP}\left(\mathrm{cm}^{-1} / \mathrm{GPa}\right) \\
\mathrm{P}>7.8 \mathrm{GPa}\end{array}$ & $\gamma$ \\
\hline $\mathrm{T}_{1 \mathrm{~g}}$ & 204.9 & $205(1)$ & 0.2 & 0.1 & $0.20(5)$ \\
$\mathrm{E}_{\mathrm{g}}$ & 309.0 & $309(1)$ & 1.9 & 0.8 & $1.25(5)$ \\
$\mathrm{T}_{1 \mathrm{~g}}$ & 468.1 & $468(1)$ & 1.7 & 1.4 & $0.74(5)$ \\
$\mathrm{T}_{1 \mathrm{~g}}$ & 563.2 & $563(1)$ & 2.0 & 1.7 & $0.72(5)$ \\
$\mathrm{A}_{1 \mathrm{~g}^{*}}$ & 613.1 & $613(1)$ & 2.2 & 2.1 & $0.73(5)$ \\
$\mathrm{A}_{1 \mathrm{~g}}$ & 688.4 & $688(1)$ & 2.4 & 2.2 & $0.71(5)$ \\
\hline \hline
\end{tabular}




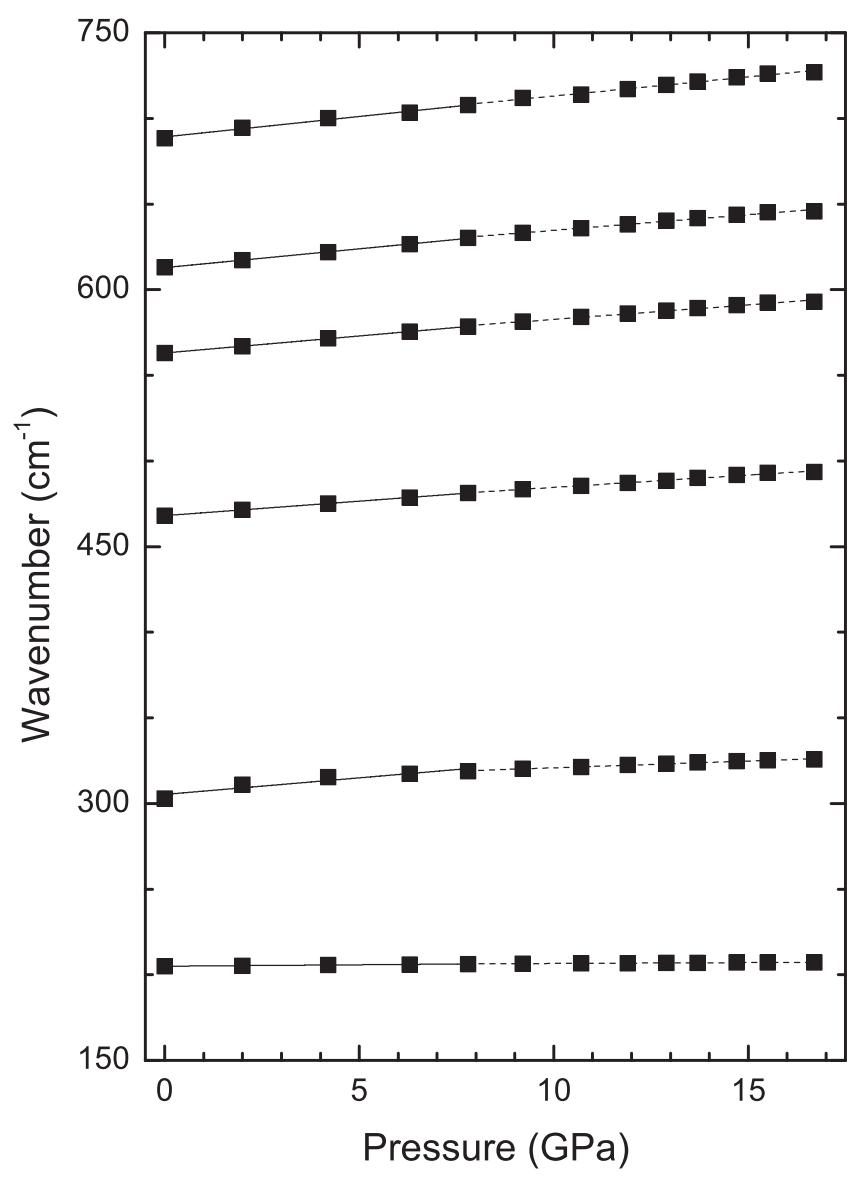

FIG. 9. Pressure dependence of Raman frequencies. Squares present results. Solid lines present linear fits for $\mathrm{P}<7.8 \mathrm{GPa}$. Dashed lines: linear fits for $\mathrm{P}>7.8 \mathrm{GPa}$.

(indicated with a line in Fig. 8). The parameter $x_{A / B}$ is equal to 0 if there is no cation inversion and equal to 1 if the cation inversion is total. In the figure, it can be seen that $x_{A / B}$ remains nearly constant below $7.8 \mathrm{GPa}$ with a value of approximately 0.17 . This means that some partial inversion is present (as determined from Mössbauer measurements at ambient pressure), but it is not affected by pressure under quasi-hydrostatic conditions. However, $\mathrm{x}_{\mathrm{A} / \mathrm{B}}$ gradually increases with pressure beyond $7.8 \mathrm{GPa}$, reaching a value of approximately 0.3 near $17 \mathrm{GPa}$. Then, under non hydrostatic conditions, cation inversion clearly increases. This conclusion is coherent with the observation previously made in spinel $\mathrm{NiAl}_{2} \mathrm{O}_{4}$, for which it has been shown that inversion can be triggered by stresses at room temperature. ${ }^{25}$ From Fig. 8, it is also clear that when the sample is decompressed, the relative intensity of the $\mathrm{A}_{1 \mathrm{~g}}$ and $\mathrm{A}_{1 \mathrm{~g}}$ * modes does not go back to its original value. In fact, $x_{A / B}$ is equal to 0.2 in the recovered sample. Thus, apparently, cation inversion is not fully recovered upon decompression. This observation coincides with the fact that the unit-cell parameter does not revert either to the ambient-pressure value. However, by the moment, we cannot establish whether this is just a mere coincidence or both facts are correlated.

The last fact we want to comment is that we did not find any evidence of the occurrence of a pressure induced phase transition in $\mathrm{CoFe}_{2} \mathrm{O}_{4}$ nanoparticles up to $17 \mathrm{GPa}$. This fact

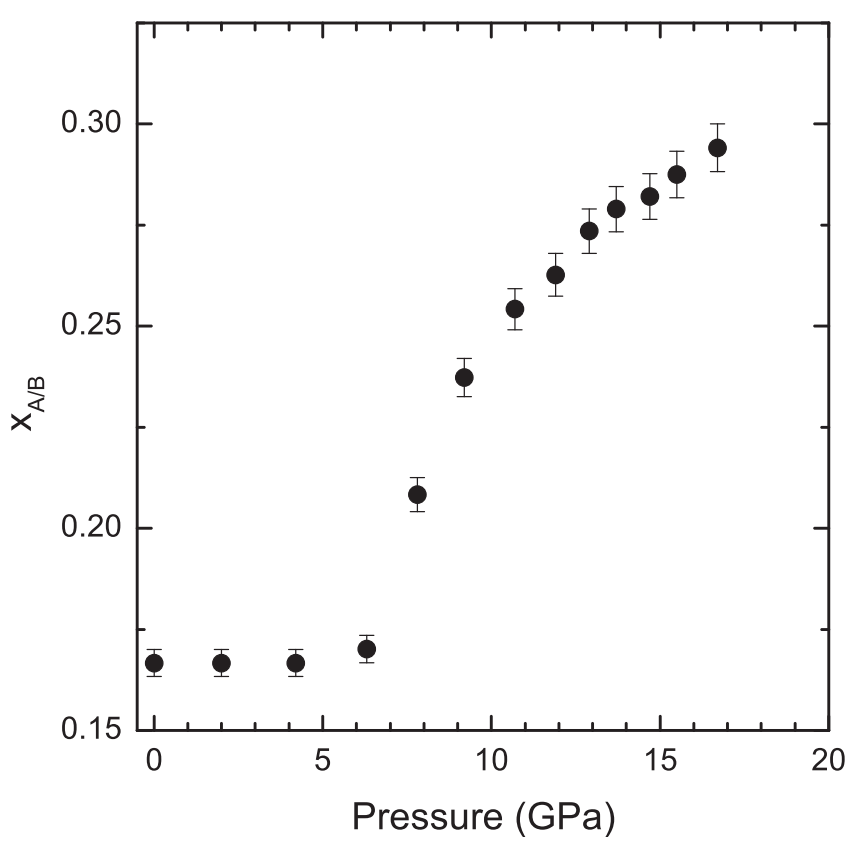

FIG. 10. $x_{A / B}=\frac{I_{A 1 g} *}{I_{A 1 g}+I_{A l g *}}$ as a function of pressure. The increase of the parameter is indicative of an increase of the cation inversion.

agrees with our XRD experiments and contradicts previous resistivity measurements. ${ }^{12}$ The agreement between our two independent experiments suggests that the resistivity changes previously reported could have been caused by changes in the electrical properties of the samples, which are not related to a structural phase transition. There are several facts that support our hypothesis. The first one is that in nanoparticles of $6 \mathrm{~nm}$, the resistivity change occurs at a pressure $(12.5 \mathrm{GPa})$ that is smaller than the transition pressure of bulk samples $(25 \mathrm{GPa})^{7}$ but higher than in the $80 \mathrm{~nm}$ particles $(7.5 \mathrm{GPa})$. This fact contradicts most of the knowledge existent on the high-pressure behavior of nanoparticles. ${ }^{13}$ According to it, one should expect the transition pressure to increase as the particle size decreases ${ }^{13}$ and should expect for the $80 \mathrm{~nm}$ larger size nanoparticles to behave similar to the bulk than the smaller size nanoparticles. We consider that other hypotheses than a structural phase transition could better explain the resistivity changes reported from HP experiments. ${ }^{12}$ The resistivity changes can be caused by percolation or tunneling effects that could easily reduce the contact resistance between grains. ${ }^{36,37}$ Both phenomena can cause conduction of electrons by hopping from one grain to a neighboring one when the interparticle distance between grains is only few nanometers, a situation that can be easily achieved by putting the sample under a pressure of several gigapascals. Additionally, the reported resistivity experiments ${ }^{12}$ could have been affected by the experimental method: two contact resistivity measurements ${ }^{38}$ and no use of PTM. ${ }^{16}$ Both things are highly not recommendable for high-pressure studies and could hinder the conclusions extracted from the experiments.

\section{CONCLUSIONS}

In this work, we report a Raman and XRD study of nanoparticles of $\mathrm{CoFe}_{2} \mathrm{O}_{4}$ under compression. The sample used for the experiments was synthesized by our group and 
characterized at ambient pressure (before HP experiment were performed) using a combination of techniques. Both HP XRD and Raman experiments indicate that, in contrast to conclusions of previous studies, $\mathrm{CoFe}_{2} \mathrm{O}_{4}$ nanoparticles remain in the cubic spinel structure up to $17 \mathrm{GPa}$. We also found that the bulk modulus resulted a $17 \%$ larger for the studied nanoparticles than for bulk $\mathrm{CoFe}_{2} \mathrm{O}_{4}$. In addition, the compression of the sample strongly depends on whether the experiments are performed under quasi-hydrostatic or non-hydrostatic conditions. On top of that we found that after decompression the lattice parameter of $\mathrm{CoFe}_{2} \mathrm{O}_{4}$ does not fully revert to the value determined before compression. From Raman spectroscopy, we determined the pressure evolution of all Raman-active phonons. These experiments also revealed irreversible changes on relative phonon intensities. We attribute this behavior to Co migration from a tetrahedral to an octahedral site in the spinel activated by deviatoric stresses, in concordance with the reordering of the mixed spinel $\mathrm{CoFe}_{2} \mathrm{O}_{4}$ nanoparticles.

\section{ACKNOWLEDGMENTS}

This work was partially funded by LNLS, Campinas Brazil (Project XDS 17704), and by ANPCyT (Project PICT 2012 1730). The authors thank L. G. Pampillo by collaboration during nanoparticle synthesis. D.E. thanks the financial support provided by the Spanish government MINECO under Grant No. MAT2013-46649-C4-1-P. The authors thank SC-SIE from Universitat de Valencia for providing HRTEM facilities.

${ }^{1}$ M. G. Brik, A. Suchocki, and A. Kaminśska, Inorg. Chem. 53, 5088 (2014). ${ }^{2}$ D. Errandonea, " $\mathrm{AB}_{2} \mathrm{O}_{4}$ compounds at high pressures," in PressureInduced Phase Transitions in $\mathrm{AB}_{2} \mathrm{X}_{4}$ Chalcogenide Compounds, Springer Series in Materials Science Vol. 189, edited by F. J. Manjón et al. (Springer-Verlag, Berlin, 2014).

${ }^{3}$ H. St. C. O'Neill and A. Navrotsky, Am. Mineral. 68, 181 (1983); available at http://ammin.geoscienceworld.org/content/68/1-2/181.short.

${ }^{4}$ R. C. Peterson, G. A. Lager, and R. L. Hitterman, Am. Mineral 76, 1455 (1991); available at http://ammin.geoscienceworld.org/content/76/9-10/ 1455.short.

${ }^{5}$ E. Greenberg, G. Kh. Rozenberg, W. Xu, R. Arielly, M. P. Pasternak, A. Melchior, G. Garbarino, and L. S. Dubrovinsky, High Pressure Res. 29, 764 (2009).

${ }^{6}$ L. W. Finger, R. M. Hazen, and A. M. Hofmeister, Phys. Chem. Minerals 13, 215 (1986).

${ }^{7}$ J. Blasco, G. Subías, J. García, C. Popescu, and V. Cuartero, J. Solid State Chem. 221, 173 (2015).
${ }^{8}$ L. Ye, S. Zhai, X. Wu, C. Xu, K. Yang, and Y. Higo, Phys. Chem. Minerals 42, 569 (2015).

${ }^{9}$ D. Errandonea, R. S. Kumar, F. J. Manjon, V. V. Ursaki, and E. V. Rusu, Phys. Rev. B 79, 024103 (2009).

${ }^{10}$ J. Darul, C. Lathe, and P. Piszora, J. Phys. Chem. C 117, 23487 (2013).

${ }^{11}$ Z. Wang, R. T. Down, V. Pischeda, R. Shetty, S. K. Saxena, C. S. Zha, Y. S. Zhao, D. Schieferl, and A. Waskowska, Phys. Rev. B 68, 094101 (2003).

${ }^{12}$ Z. Wu, Z. Bao, L. Cao, C. Liu, Q. Li, S. Xe, and B. Zou, J. Appl. Phys. 93, 9983 (2003).

${ }^{13}$ A. San Miguel, Chem. Soc. Rev. 35, 876 (2006).

${ }^{14}$ C. Popescu, J. A. Sans, D. Errandonea, A. Segura, R. Villanueva, and F. Sapinã, Inorg. Chem. 53, 11598 (2014).

${ }^{15}$ O. Gomis, J. A. Sans, R. Lacomba-Perales, D. Errandonea, Y. Meng, J. C. Chervin, and A. Polian, Phys. Rev. B 86, 054121 (2012).

${ }^{16}$ D. Errandonea, A. Muñoz, and J. Gonzalez-Platas, J. Appl. Phys. 115, 216101 (2014).

${ }^{17}$ D. Errandonea, Y. Meng, M. Somayazulu, and D. Haüsermann, Physica B 355, 116 (2005)

${ }^{18}$ A. Veiga, M. A. Mayosky, N. Martínez, P. Mendoza Zélis, G. A. Pasquevich, and F. H. Sánchez, Hyp. Int. 202, 107 (2011).

${ }^{19}$ H. K. Mao, J. Xu, and P. M. Bell, J. Geophys. Res. 91, 4673, doi:10.1029/ JB091iB05p04673 (1986).

${ }^{20}$ S. Klotz, J. C. Chervin, P. Munsch, and G. Le Marchand, J. Phys. D 42, 075413 (2009).

${ }^{21}$ L. Lutterotti, M. Bortolotti, G. Ischia, I. Lonardelli, and H.-R. Wenk, Z. Kristallogr. 26, 125 (2007).

${ }^{22}$ J. D. Baraliya and H. H. Joshi, Vib. Spectrosc. 74, 75 (2014).

${ }^{23}$ L. Kumar, P. Kumar, A. Narayan, and M. Kar, Int. Nano Lett. 3, 8 (2013).

${ }^{24}$ B. D. Cullity and S. R. Stock, Elements of X-Ray Diffraction, 3rd ed. (Prentice-Hall Inc., 2001), pp. 167-171.

${ }^{25}$ I. Halevy, D. Dragoi, E. Üstündag, A. F. Yue, E. H. Arredondo, J. Hu, and M. Somayazulu, J. Phys.: Condens. Matter 14, 10511 (2002).

${ }^{26}$ D. Errandonea, C. Ferrer Roca, D. Martinez Garcia, A. Segura, O. Gomis, A. Muñoz, P. Rodriguez-Hernandez, J. Lopez-Solano, S. Alconchel, and F. Sapiña, Phys. Rev. B 82, 174105 (2010).

${ }^{27}$ F. Birch, J. Geophys. Res. 57, 227, doi:10.1029/JZ057i002p00227 (1952).

${ }^{28}$ R. J. Angel, J. Gonzalez-Platas, and M. Alvaro, Z. Kristallogr. 229, 405 (2014).

${ }^{29}$ N. Hansen, Scr. Mater. 51, 801 (2004).

${ }^{30}$ A. B. Garg, D. Errandonea, P. Rodríguez-Hernández, S. López-Moreno, A. Muñoz, and C. Popescu, J. Phys.: Condens. Matter 26, 265402 (2014).

${ }^{31}$ A. Garg and D. Errandonea, J. Solid State Chem. 226, 147 (2015).

${ }^{32}$ L. Malavasi, C. Tealdi, M. Amboage, M. C. Mozzati, and G. Flor, Nucl. Instrum. Methods Phys. Res. B 238, 171 (2005).

${ }^{33}$ P. Chandramohan, M. P. Srinivasan, S. Velmurugan, and S. V. Narasimhan, J. Solid State Chem. 184, 89 (2011).

${ }^{34}$ M. A. Laguna-Bercero, M. L. Sanjuan, and R. I. Merino, J. Phys.: Condens. Matter 19, 186217 (2007).

${ }^{35}$ D. Errandonea, R. Boehler, S. Japel, M. Mezouar, and L. R. Benedetti, Phys. Rev. B 73, 092106 (2006)

${ }^{36}$ C. Li, E. T. Thostenson, and T.-W. Chou, Appl. Phys. Lett. 91, 223114 (2007).

${ }^{37}$ A. Buldum and P. L. Jian, Phys. Rev. B 63, 161403 (2001).

${ }^{38}$ D. Errandonea, A. Segura, D. Martínez-García, and V. Muñoz-San Jose, Phys. Rev. B 79, 125203 (2009). 